\title{
The use of synthetic foods to optimise microwave-assisted digestion procedures
}

\author{
W. Zorgati, D.N. Rutledge and M.H. Feinberg* \\ Institut National de la Recherche Agronomique, Laboratoire de Chimie Analytique 16, rue Claude Bernard, \\ 75231 Paris Cedex 05, France
}

\begin{abstract}
Major food constituents such as proteins, fats and carbohydrates are generally recognised as having a strong influence on the performance of acid sample digestion. Various Scheffé mixture experimental designs were used to detect the influence and the interactions between these constituents during the Kjeldahl nitrogen digestion using focused microwaves. Pure ingredients were mixed in different proportions in order to prepare 7 synthetic food samples covering a wide range of foods types. 3 heating powers were applied and nitrogen recovery yields were recorded at different times. Two types of models were proposed to describe the data: one that explains the shape of the recovery profiles, the other that gives the response surfaces of the recovery yield as a function of digestion time. From these models precise guidelines to optimise the digestion program of a given food can be deduce.
\end{abstract}

Keywords. Microwave - Kjeldahl - digestion - mixture design.

\section{Introduction}

Sample digestion still remains a limiting factor in food control. It is time-consuming and widely based on empirical knowledge. Over the last decade, several substitutive techniques have been proposed to reduce the digestion time or to automate the procedure. In that respect, microwave heating is rather successful as it usually dramatically reduces the digestion time. However it requires an intensive experimental step to define the optimal operating conditions because microwave-assisted procedures are very different from the earlier procedures they are supposed to replace and most of the prior "know-how" of the analysts is therefore obsolete. This study presents an interesting approach to define general guidelines for optimising microwave-assisted digestion procedures.

The procedure is illustrated using the determination of proteins in foods. Kjeldahl's method is a widely-used reference method for total nitrogen determination in food products [1]. The success of this more than 100-year old technique can be explained by its simplicity as it contains just three steps: digestion, distillation and titration. The digestion consists in destroying organic matter with concentrated sulphuric acid, which quantitatively oxidises protein nitrogen into ammonium bisulphate. This destruction occurs in boiling sulphuric acid for 1 or 2 hours in most cases. Distillation and titration are done in automated devices specially designed for food analysis.

On the one hand, for some samples it is necessary to complete the oxidation by the adding powdered potassium permanganate as auxiliary oxidant [2]. On the other hand, many reference techniques propose to add a "catalyst" in order to increase the boiling point of the sulphuric acid and facilitate the destruction of organic matter.

For most food samples it has been demonstrated that using a microwave heating technique seriously decreases the duration of the digestion step. This reduction is mainly due to the microwave energy transfer, which goes from the reagent medium towards the external cavity of the digester, whereas traditional heating consists in heating of the reaction vessel and transfer of the thermal energy to the reagent medium by conduction. Thus, the thermic yield of a microwave heating is much more efficient. At the same time, it is possible to avoid the use of a catalyst by adding hydrogen peroxide in the final step of the digestion. Thus, a typical food digestion procedure consists of two complementary periods: the charring which is early decomposition of the organic matter in pure sulphuric acid; and the oxidation with hydrogen peroxide, to destroy resistant molecules or molecular bounds [3].

Several optimisation studies have been carried out on the basis of this procedure. The use of experimental design methodology has been very relevant to selecting the bestadapted operating conditions. A validation by interlaboratory analysis of focused microwaves digestions have even been conducted [4].

As a general conclusion for these studies, it has become clear that the important variability of food matrices makes difficult the definition of a common procedure applicable to all foods. Although it is possible to define large groups of 


\section{Original articles}

matrices presenting a similar behaviour, exceptions are abundant. Optimal digestion programs must be adjusted to the specificity of the different food products and the choice of heating times and heating powers are sometimes made case by case.

It is well known that the proximate constituents of a food, such as total fat, protein, total carbohydrates, ash, and moisture, widely determine its chemical behaviour during acid digestion. When considering the Kjeldahl digestion technique, the variations in ash content, while potentially affecting trace element determinations, can be considered as having a minor influence. Similarly, moisture content, while influencing many analytical procedures, has negligible effect here. Finally, only 3 major constituents - fat, protein and carbohydrates - were retained as having an influence on the performances of Kjeldahl digestion. Therefore, it is possible to propose a classification of foods, in relation to their behaviour during digestion, based on their relative contents in fat, protein and carbohydrates in dry matter [5].

In this study a reduced set of synthetic food samples (SFS) was prepared by mixing three "pure" food products presenting high concentrations of proteins, carbohydrates and fats. The aim was to detect the influence of these constituents on the efficiency of the charring step. The mixture design technique was used in order to obtain predictive mathematical models. These mathematical models give a global and continuous representation of the ability to destroy food matrices in presence of sulphuric acid when using microwaves.

\section{Methods and experimental}

\section{Mixture design methodology}

Mixture designs can be used when the experimental factors are dependent in such a way that the sum of all applied levels is constant (usually set to 1.0 or $100 \%$ ) [6]. Moreover, the experimental response is assumed to depend only on the relative proportions of the ingredients present in the mixture and not on the amount of the mixture. The total amount is held constant and the value of the response changes when changes are made in the relative proportions of ingredients put in the mixture. The response is said to be a measure of the joint blending property of the ingredients in the mixture.

In this study, a three-component simplex-centroïd design proposed by Scheffé was selected because constraints can be applied to the experimental domain. In the present case, all synthetic food samples (SFS) contain at least $10 \%$ of proteins in order to satisfy the following criteria:

- the SFS must be representative of most food products according to their proportions in proteins, carbohydrates and fats; it was verified that about $75 \%$ of existing food products are contained within this reduced experimental domain [7];
- each SFS must contain at least a few milligrams of nitrogen, in order to be above the detection limit of the technique.

For three factors, this design, also called special cubic design, consisted of 7 trials. The selected experimental points and the relative proportions of the ingredients in the SFS are represented in figure 1 . For each point, the response was measured and a polynomial additive model fitted between the response and the levels of factors. In order to improve the estimation of the experimental error, measurements were replicated and 14 trials were collected, giving 7 degrees of freedom to compute the residual error.

With such an experimental design it was possible to use a third-degree response surface model as described by (1). The number of coefficients in the model was the same as the number of points in the design, leading to very high design efficiency. The canonical polynomial equation of the response surface is:

$$
\begin{gathered}
y=b_{1} x_{1}+b_{2} x_{2}+b_{3} x_{3}+b_{12} x_{1} x_{2} \\
+b_{13} x_{1} x_{3}+b_{23} x_{2} x_{3}+b_{123} x_{1} x_{2} x_{3}+e .
\end{gathered}
$$

In this equation, $y$ is the observed response (i.e. the Kjeldahl nitrogen recovery); $x_{1}, x_{2}, x_{3}$ are the proportions of the ingredients in the mixture, respectively proteins, carbohydrates and fats; $b_{1}, b_{2}, b_{3}, b_{12}, b_{13}, b_{23}, b_{123}$ are the estimated coefficients of the model and $e$ the residual experimental error.

The estimated coefficients were calculated by Multiple Linear Regression. Experimental design processing and data analyses were performed using Statgraphics Plus (Manugistics) [8]. The standard errors of the estimated

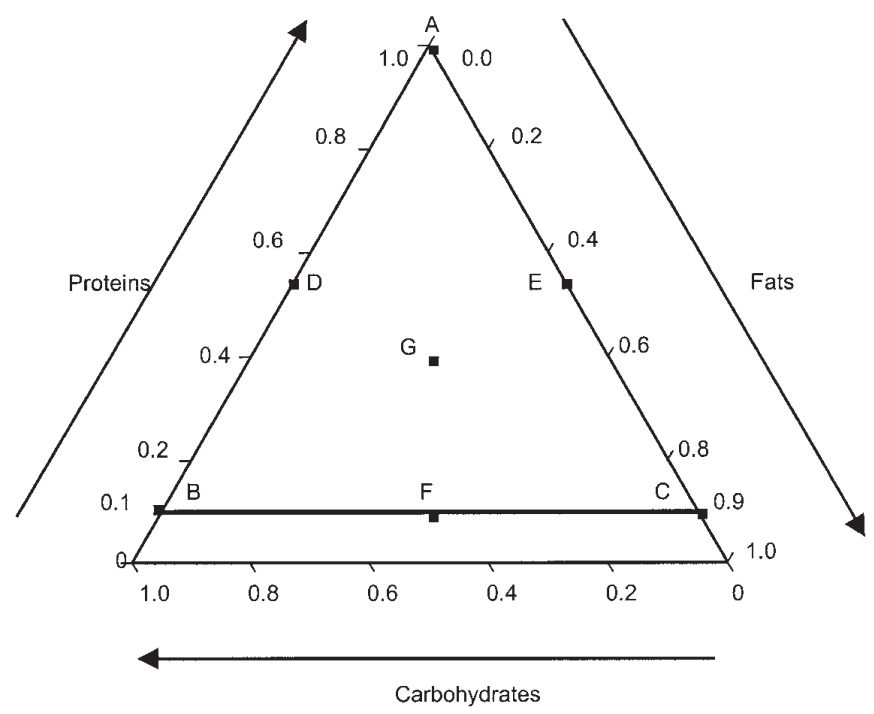

Figure 1. The Scheffé mixture design giving the theoretical composition of the SFS. Each SFS is indicated by an letter ranging from $\mathrm{A}$ to $\mathrm{G}$. 
coefficients are proportional to $s_{\mathrm{e}}^{2}$, the estimation of the residual variance.

In a Scheffé simplex-centroïd design, the experimental domain is a 2-dimensional regular simplex, which can be represented by an equilateral triangle (see figure 1). Samples A, B, C correspond to the linear terms; samples D, E, F correspond to the rectangular terms; sample $\mathrm{G}$ corresponds to the cubic term [9].

On the other hand, the appearance of discontinuities or strong variations in the limits of the experimental field may perturb the results and lead to outlying effects. The goodness of fit of the regression equation was measured by the multiple correlation coefficient, $r$. The square of the multiple correlation coefficient $r^{2}$ links the observed response to the pattern estimated response. The $r^{2}$ associated with the fit of Scheffé-type canonical polynomials (with no constant term) is inflated. As a result, an adjusted $r^{2}$ statistic () has been suggested when fitting the canonical polynomials [6].

\section{Preparation of synthetic food samples}

For the preparation of the synthetic food samples, three "pure" ingredients were used as assumed to contains a very high contents of each constituent: bovine albumin for proteins, sucrose for carbohydrates, and sunflower oil for fats.

SFS preparation consisted in weighing the proportions of each ingredient so that the total weight of the blend was $0.5 \mathrm{~g}$. Then $1.5 \mathrm{ml}$ of water was added in order to improve the homogeneity of the mixture. This was done with a Vortex ${ }^{\mathrm{TM}}$ blender for 10 to 15 minutes. If the blend was not yet homogeneous (i.e. some components or aggregates remain visible), the operation was continued with magnetic stirrers.

\section{Measurement of the nitrogen recovery}

Microwave digestion was performed at atmospheric pressure in a Maxidigest ${ }^{\mathrm{TM}}$ MX-350 (Prolabo). This device is composed of a focused microwave generator with a maximum power of $300 \mathrm{~W}$ controlled by a programmer. Two glass beads were also placed in each digestion flask to regulate boiling and reduce foaming. The different heating powers were expressed as a percentage of the maximum power. The duration of each step was expressed in minutes.

Many studies have shown that food composition largely affect the efficiency of the charring step. This study was focused on the charring step and several different mixture designs were used under varying conditions of heating power and digestion charring time. Three different heating powers were used: $30 \%, 45 \%$ and $60 \%$. For each of them the nitrogen recovery was monitored over 20 minutes and the nitrogen contents measured every 2 minutes. Therefore, 10 replicated mixture designs using the 7 pattern SFS and corresponding to the different pairs heating power (\%) and time (min) were achieved.

After cooling, the digestion vessel it was directly installed in a Kjeltec ${ }^{\mathrm{TM}} 1026$ (Perstorp) distilling unit for $\mathrm{NH}_{3}$ titration according to the classical Kjeldahl procedure. Recovered nitrogen amounts were calculated according to specifications of the Afnor standard [1] and expressed as a percent of the theoretical nitrogen concentration of the SFS. This theoretical nitrogen concentration could be directly calculated from the ingredient composition of the SFS.

\section{Results and discussion}

\section{Nitrogen recovery profiles as a function of time and power}

The nitrogen recovery yields of all the different samples are calculated with 2 replicates. In figure 2, the individual profiles of the SFS at $30 \%$ heating power are presented. Samples B, C, and F contain the same amounts of proteins; only the amounts of carbohydrates and fats change. However, the nitrogen recovery yields of these three samples do not vary in the same way during the digestion. The same remark can be made for samples D and E - their nitrogen recovery yields do not evolve similarly during the digestion. From these remarks it can be assumed that carbohydrates and fats have a strong influence on the process of digestion at this power heating. On the other hand, the evolution of the nitrogen recovery yields at heating powers $45 \%$ (Fig. 3) and $60 \%$ (Fig. 4) for samples with the same amounts of proteins also seem to vary in different ways

\begin{tabular}{ccc}
\hline $\begin{array}{c}\text { Nutriment } \\
(\text { brand })\end{array}$ & $\begin{array}{c}\text { Ingredient used } \\
(\mathrm{g} / 100 \mathrm{~g})\end{array}$ & Theoretical concentration \\
\hline Proteins & Bovine albumin - fraction V \\
Total carbohydrates & (Ref. 04-10-810B from Euromedex $)$ & 16.14 \\
Total fats & Kectapur $(+)$ Sucrose & 99.97 \\
Commercial Sunflower oil & 99.99 \\
\hline
\end{tabular}




\section{Original articles}

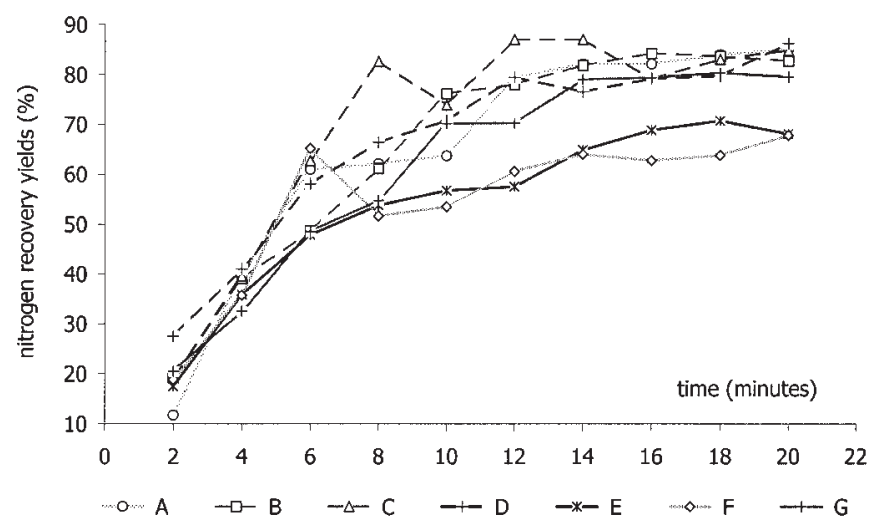

Figure 2. Individual synthetic food sample recovery profiles at $30 \%$ of the heating power.

when compared to the heating power $30 \%$. Thus, the effects of carbohydrates and fats seem to be essential.

In general, at the beginning of the digestion, the nitrogen recovery yield evolves in an exponential way and tends to become constant after a few minutes. It was possible to fit the resulting profiles with the following empirical model:

$$
y_{\mathrm{HP}, \mathrm{SFS}}=a_{0}-\exp \left(a_{1}-a_{2} \times t\right)
$$

where: $y_{\mathrm{HP}, \mathrm{SFS}}$ is the observed response for a given heating power $(H P)$ and synthetic food sample $(S F S) ; t$ the digestion time; $a_{0}$ the nitrogen recovery yields at the end of the profile; $a_{1}$ the nitrogen recovery yields at the beginning and $a_{2}$ the digestion rate constant. An in-house program [10] based on the Marquardt non-linear regression algorithm [11] was used to estimate the coefficients of this model for each SFS and heating power. The values of $r_{\mathrm{A}}^{2}$ associated with the fitted models are recorded in table I. They are all higher than $80 \%$ for all food sample models and for each power heating. In order to illustrate the typical quality of the model, the responses predicted with the model computed for mixture $\mathrm{D}$ at heating power $45 \%$ are compared with the experimental values. These results are illustrated by figure 5 . The corresponding $r_{\mathrm{A}}^{2}$ is equal to 0.990 and the calculated model is:

$$
y_{45, \mathrm{D}}=84.52-\exp (4.84-0.44 \times t) .
$$

The values of the different coefficients and their associated standard errors are represented in table I. According to the values of coefficient $a_{0}$, it appears the heating power has little effect on the nitrogen recovery yield at the end of the digestion, for foods having only proteins (sample A) or presenting comparable amounts of proteins and carbohydrates (sample D) or presenting comparable amounts of proteins, fats and carbohydrates (sample G). When carbohydrates are absent (sample C), the medium heating power $(45 \%)$ seems more favourable. Finally, when there is a lot of

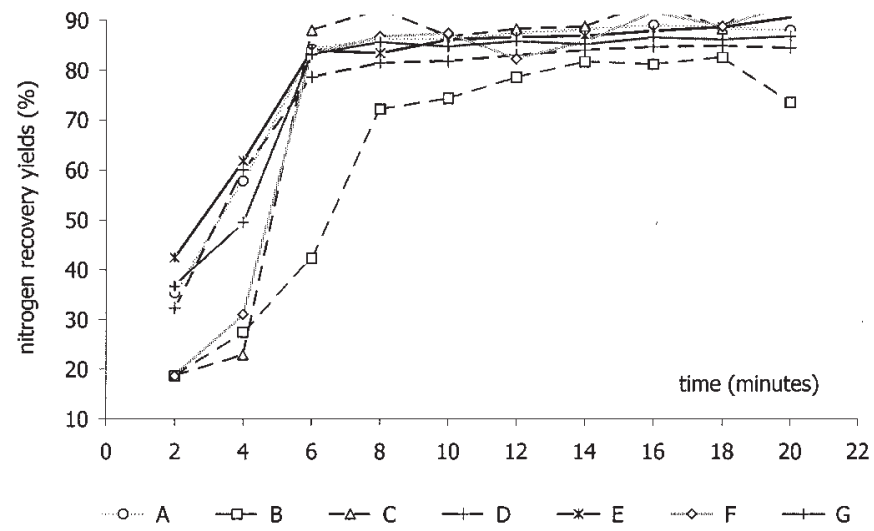

Figure 3. Individual synthetic food sample recovery profiles at $45 \%$ of the heating power.

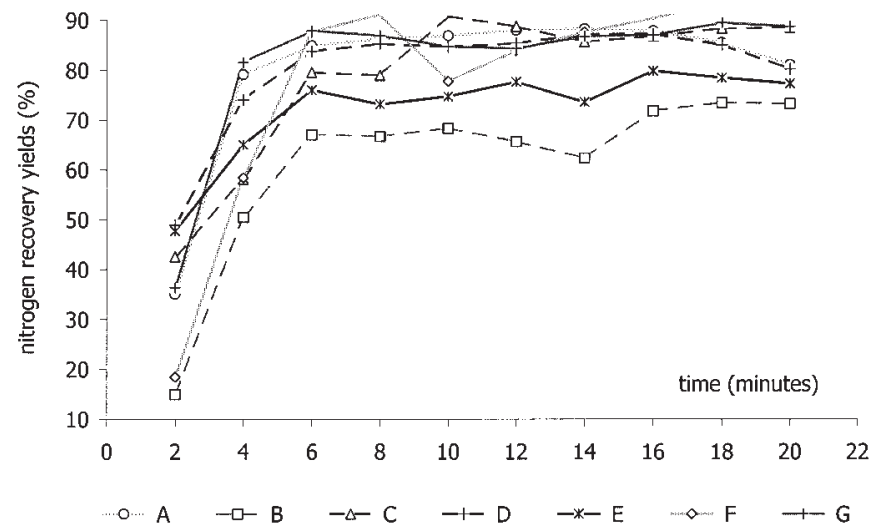

Figure 4. Individual synthetic food sample recovery profiles at $60 \%$ of the heating power.

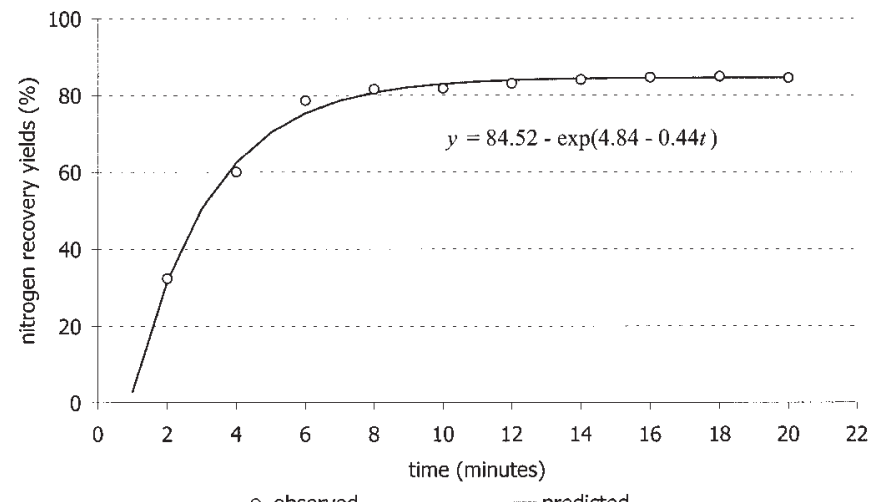

Figure 5. Observed and predicted recovery profiles for synthetic sample $\mathrm{D}$, at $45 \%$ of the heating power. 
Table I. Coefficients of the profile evolution models for each SFS and heating power. (Standard error of coefficients are given between parentheses).

\begin{tabular}{|c|c|c|c|c|c|}
\hline Heating power (\%) & $S F S$ & $a_{0}\left(s_{a 0}\right)$ & $a_{1}\left(s_{a l}\right)$ & $a_{2}\left(s_{a 2}\right)$ & $r_{A}^{2}$ \\
\hline 30 & A & $85.830(2.4)$ & $4.702(0.1)$ & $0.210(0.0)$ & 0,963 \\
\hline 45 & A & $89.064(1.1)$ & $4.810(0.1)$ & $0.399(0.0)$ & 0,963 \\
\hline 60 & A & $86.188(0.7)$ & $5.847(0.3)$ & $0.969(0.1)$ & 0,966 \\
\hline 30 & B & $89.354(2.5)$ & $4.607(0.0)$ & $0.170(0.0)$ & 0,976 \\
\hline 45 & B & $85.122(4.6)$ & $4.674(0.1)$ & $0.196(0.0)$ & 0,892 \\
\hline 60 & B & $69.443(1.1)$ & $5.135(0.2)$ & $0.567(0.1)$ & 0,949 \\
\hline 30 & $\mathrm{C}$ & 85.849 (3.6) & $4.806(0.2)$ & $0.283(0.1)$ & 0,850 \\
\hline 45 & C & $95.135(4.8)$ & $5.031(0.2)$ & $0.300(0.1)$ & 0,812 \\
\hline 60 & $\mathrm{C}$ & 88.478 (1.4) & $4.886(0.1)$ & $0.391(0.0)$ & 0,948 \\
\hline 30 & D & $85.529(2.1)$ & $4.441(0.0)$ & $0.180(0.0)$ & 0,971 \\
\hline 45 & D & $84.517(0.5)$ & $4.842(0.1)$ & $0.439(0.0)$ & 0,990 \\
\hline 60 & D & $85.156(0.6)$ & $4.876(0.2)$ & $0.640(0.1)$ & 0,961 \\
\hline 30 & $\mathrm{E}$ & 70.943 (1.8) & $4.314(0.0)$ & $0.180(0.0)$ & 0,972 \\
\hline 45 & $\mathrm{E}$ & $88.739(1.0)$ & $4.605(0.1)$ & $0.375(0.0)$ & 0,962 \\
\hline 60 & $\mathrm{E}$ & $76.688(0.9)$ & $5.320(0.2)$ & $0.723(0.1)$ & 0,951 \\
\hline 30 & $\mathrm{~F}$ & $63.352(2.5)$ & $4.492(0.2)$ & $0.344(0.1)$ & 0,801 \\
\hline 45 & $\mathrm{~F}$ & $91.524(3.5)$ & $4.986(0.2)$ & $0.314(0.1)$ & 0,871 \\
\hline 60 & $\mathrm{~F}$ & $89.066(1.9)$ & $5.617(0.3)$ & $0.679(0.1)$ & 0,909 \\
\hline 30 & $\mathrm{G}$ & $87.925(2.9)$ & $4.527(0.0)$ & $0.142(0.0)$ & 0,978 \\
\hline 45 & G & $87.646(1.9)$ & $4.692(0.1)$ & $0.352(0.1)$ & 0,903 \\
\hline 60 & $\mathrm{G}$ & $86.942(0.6)$ & $6.217(0.4)$ & $1.146(0.2)$ & 0,974 \\
\hline
\end{tabular}

Table II. Values of the adjusted coefficients of determination, $r_{A}^{2}$, of the response surface models as a function of time and heating power.

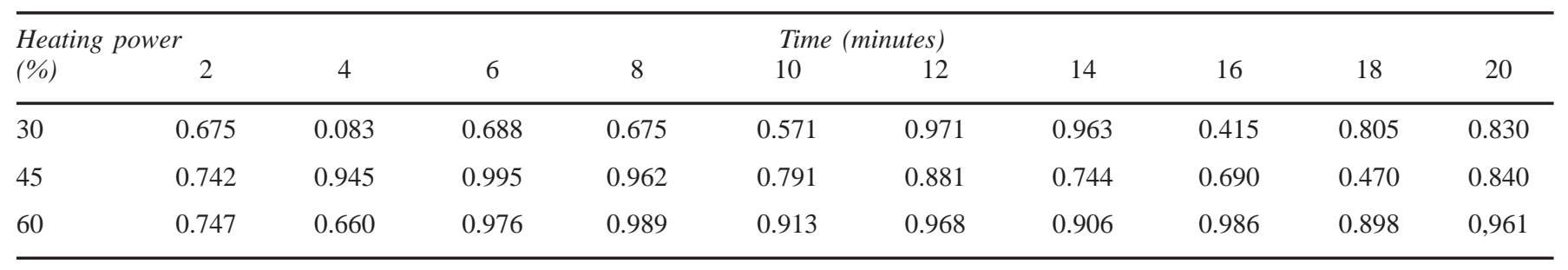

carbohydrates (sample B), the highest heating power (60\%) gives the lowest results, and when the amounts of carbohydrates and fats are equivalent (sample F), the lowest heating power $(30 \%)$ gives the lowest results.

Coefficient $a_{1}$, representing the nitrogen recovery yields at the beginning of the digestion, and coefficient $a_{2}$, representing the digestion rate constant, present similar variations as they seem to increase as a function of the heating power, for most SFS. It is not surprising to show that the rate of digestion is dependent on the heating power, but its value is also related to the matrix composition. Higher rates are observable for samples A and $\mathrm{G}$, while the lowest is for sample $\mathrm{C}$ (rich in fats and poor in carbohydrates).

\section{Response surfaces}

Response surface models (see equation (1)) were fitted to the 30 different mixture designs corresponding to the 3 heating powers and 10 digestion times. A global evaluation of the fit is given by the values of $r_{\mathrm{A}}^{2}$, reported in table II. Most of these values are above $70 \%$ indicating that the proposed model is feasible. Three rather low values are present: $8.30 \%$ for heating power $30 \%$ at 4 minutes, $41.47 \%$ for heating power $30 \%$ at 16 minutes, and $46.98 \%$ for heating power $45 \%$ at 18 minutes. No explanation could be found for these "outlying" values.

An example of the response surfaces which are obtained by using the model is shown at figure 6 . It corresponds to the nitrogen recovery yield model at 12 minutes for a heating power of $60 \%$. Its equation is given by:

$$
\begin{aligned}
\hat{y}= & 87.9 x_{1}+65.6 x_{2}+88.7 x_{3}+34.2 x_{1} x_{2} \\
& -42.8 x_{1} x_{3}+27.0 x_{2} x_{3}+40.0 x_{1} x_{2} x_{3}
\end{aligned}
$$

This surface shows that high nitrogen recovery yields are obtained when the product is rich in proteins or fats. When fats and proteins are at comparable concentrations, the recovery yield is decreased. But the lowest values are obtained when the samples are rich in carbohydrates. These 


\section{Original articles}

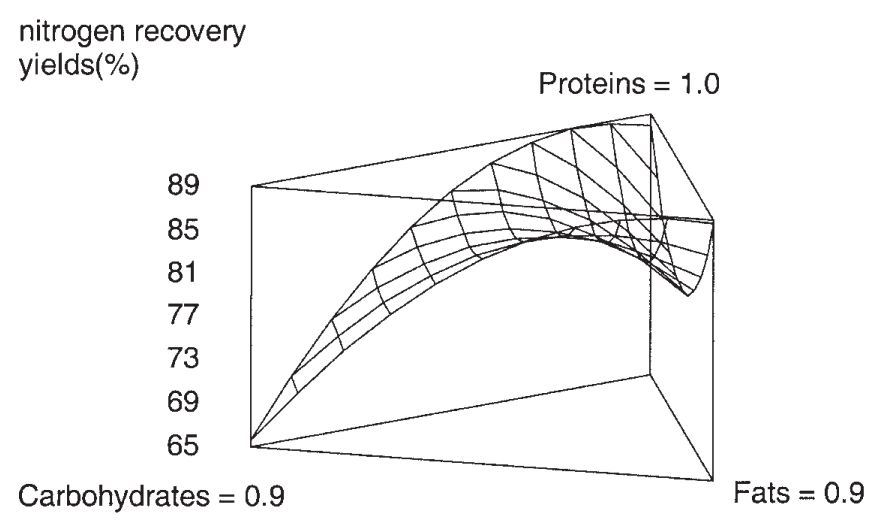

Figure 6. Nitrogen recovery yield response surface after $12 \mathrm{~min}$ utes of digestion, at $60 \%$ of the heating power.

results roughly confirm the importance of the interactions observed with the profile models.

In order to have more precise interpretation rules, and given the large number of possible response surfaces, it was easier to try to directly interpret the values of the model coefficients. The first step was to select only the significant coefficients. A classical Student- $t$ test was applied for all coefficients with a confidence level of $95 \%$. Then, the interpretation was focused on the interaction coefficients because they can help to understand the relative role of the matrix components in regard of the digestion rate. Figure 7 to figure 10 present the values of the 2 -order and 3-order interactions for the various heating powers at different digestion times.

When the heating power is set to $30 \%$ (Fig. 7), the interactions proteins-fats and carbohydrates- fats have negative effects on nitrogen recovery measurements from the $8^{\text {th }}$ minute on. The interactions proteins-carbohydrates have no influence but the positive effect of the 3-order interactions is visible at the $10^{\text {th }}$ minute. Therefore, a low heating power may be troublesome after the $8^{\text {th }}$ minute for foods that contain fats alone or combined with one of the other factors of the blend. But, they have the opposite effect as soon as they are combined with all other factors.

When considering the values of interaction coefficients for a heating power of $45 \%$ (Fig. 8), it can observed that, from the $8^{\text {th }}$ minute, most of the interactions seem to have no influence on the digestion. Applying this heating power could be recommended in order to develop robust standard programs. The interaction proteins-fats does not show any influence on the digestion when using a heating power of $45 \%$ although it tends to have a negative influence when applying higher or lower heating powers.

For a heating power of $60 \%$ (Fig. 9), the interactions proteins-carbohydrates and fats- carbohydrates have a positive effect on the digestion efficiency, while the interaction

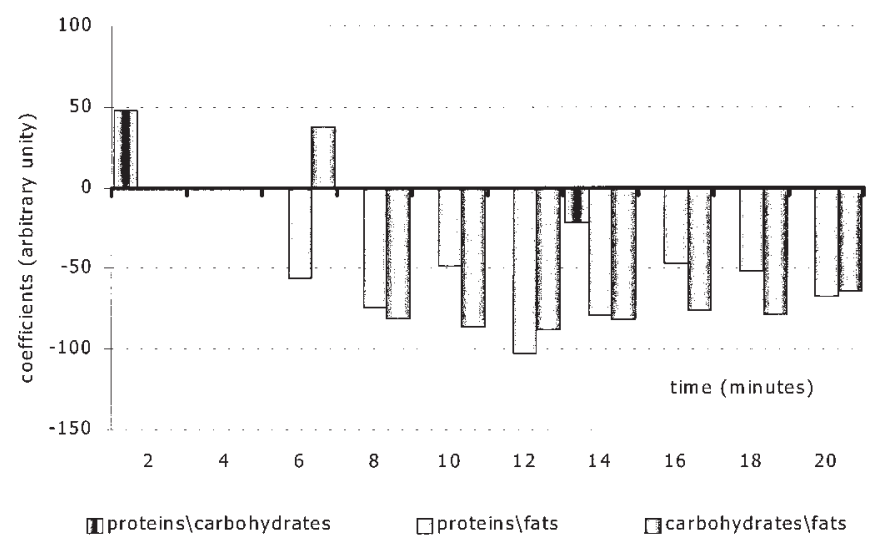

Figure 7. 2-factor interactions coefficients at heating power $30 \%$ for each digestion time. Only significant coefficients are presented.

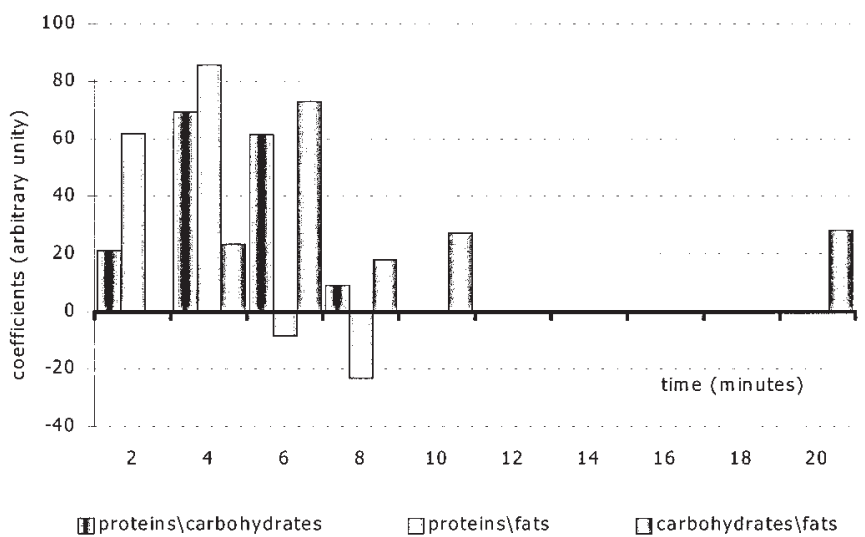

Figure 8. 2-factor interactions coefficients at heating power $45 \%$ for each digestion time. Only significant coefficients are presented.

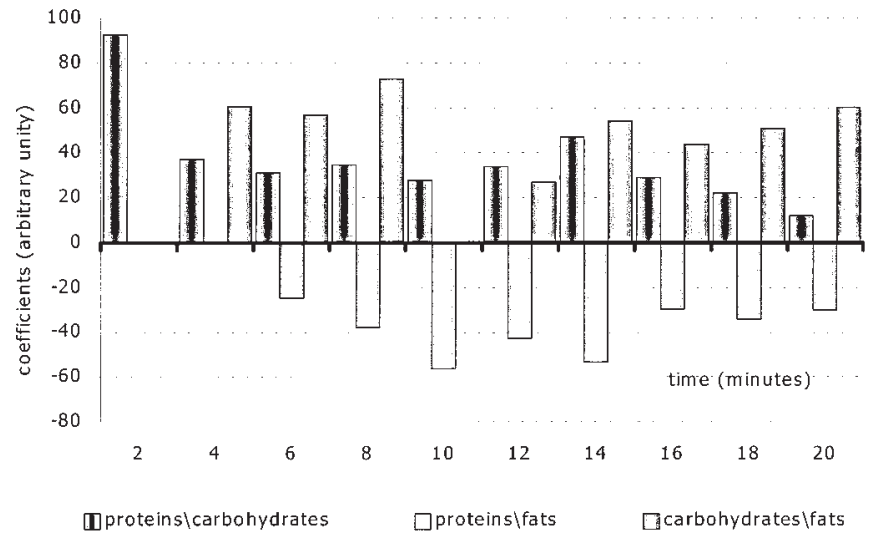

Figure 9. 2-factor interactions coefficients at heating power $60 \%$ for each digestion time. Only significant coefficients are presented. 


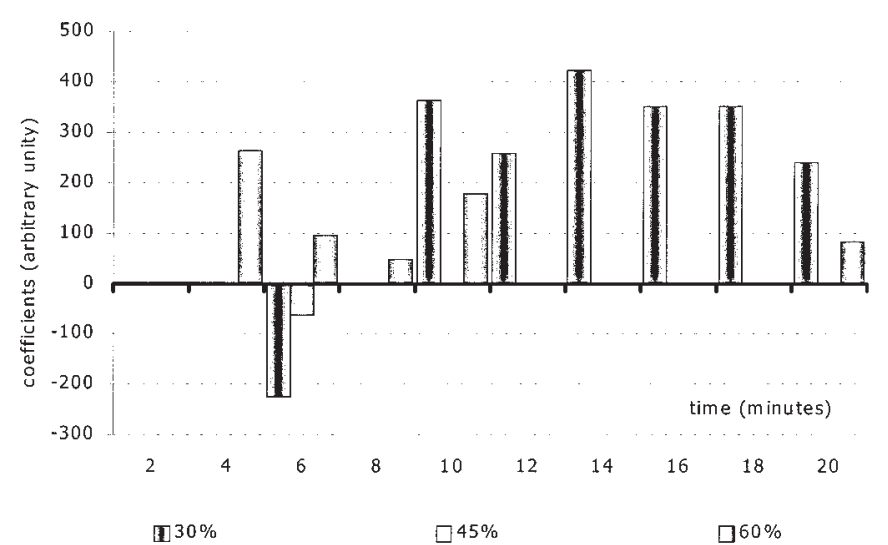

Figure 10. 3-factor interactions coefficients for each digestion time. Only significant coefficients are presented.

proteins-fats has a negative effect. The 3 -factor interaction does not show any particular influence. Thus, the effect of fats combined to other factors is interesting at this heating power because its interaction positively influences the digestion, although, the digestion is inhibited at the $6^{\text {th }}$ minute when there is no fats.

The 3-order interaction (Fig. 10) has a positive effect only in the beginning of the digestion procedure at heating power $60 \%$ or after 10 minutes using heating power $30 \%$. Some complementary remarks may help to design the best-adapted digestion program depending on the matrix composition. The interaction proteins-carbohydrates have no effect on the digestion at low heating powers but a positive effect appears when using the highest heating power. The interaction carbohydrates-fats is more variable but seems to have a negative effect at $30 \%$, becomes positive at the $10^{\text {th }}$ minute for $45 \%$ power heating and remains positive during all the digestion time for a power heating of $60 \%$.

Using simultaneously all these models, it is possible to define the best adapted conditions as a function of an actual sample. However, a global verification was performed by drawing the recovery profiles of two samples presenting a composition close to that of two SFS used in the mixture design, respectively powdered egg $(\mathrm{N} \approx 7.7 \mathrm{~g} / 100 \mathrm{~g})$ for sample $B(N \approx 8.9 \mathrm{~g} / 100 \mathrm{~g})$ and rice $(\mathrm{N} \approx 1.4 \mathrm{~g} / 100 \mathrm{~g})$ for sample $\mathrm{E}(\mathrm{N} \approx 1.6 \mathrm{~g} / 100 \mathrm{~g})$. The nitrogen contents of these two real samples was very accurately known because they have been used in a collaborative study. The results are reported in figure 11, showing a rather good adequacy between the profiles. This gives some argument to the validation of this approach based on synthetic food samples to define digestion procedures.

\section{Conclusion}

Not surprisingly, this study confirms the strong influence of the food sample proximate composition on the Kjeldahl

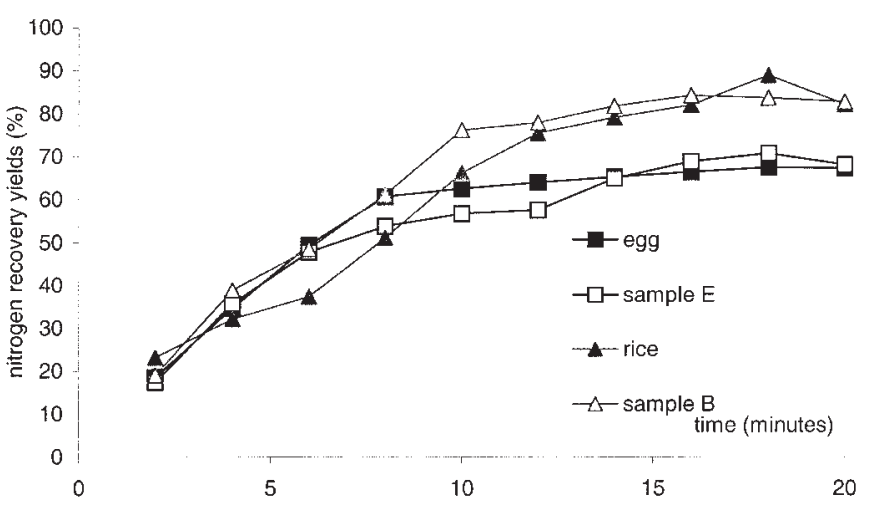

Figure 11. Comparison of the recovery profiles for two actual samples and the corresponding synthetic food samples.

nitrogen digestion efficiency. More surprisingly, it shows that the effects of the 3 major constituents of the matrix may have a very subtle influence depending on either their total or relative concentrations.

The use of synthetic food samples prepared according to an experimental design is a very convenient technique for understanding these complex relations. It can be considered as comparable to a standard addition technique performed with the components of the matrix instead of the analyte. Therefore, all criticisms put forward against the standard addition technique can also be listed for SFS: the "speciation" of the components used in the blend is different from their actual chemical forms when they are present in real food samples, the chemical interactions may be more complex than those obtained by a simple blending... These remarks are true but, in the absence of sufficient certified reference samples to cover the wide variety of food matrices, this approach gives very consistent and relevant indications. Moreover, it is an attempt to give quantitative indications on the role of the matrix during sample preparation. It should be easy to generalise this procedure to other sample types and/or analytes.

The economic importance of sample preparation in analytical chemistry may justify the need to find a more rapid and efficient methodology in order to develop operating procedures. It is well established that experimental designs are one of the elements of this strategy. The use of mixture designs to prepare artificial samples is simply an extension of this.

\section{References}

1. Norme NF V03-050, Agricultural Food Products; General Directions for the Determination of Nitrogen by the Kjeldahl method, Paris: AFNOR, 1975.

2. McKenzie, H. A. Trends in Analytical Chemistry 1994, 13, (4), 138. 


\section{Original articles}

3. Feinberg, M. H.; Ireland-Ripert, J.; Mourel, R. M. Anal. Chim. Acta 1993, 272, 83-90.

4. Suard, C.; Feinberg, M. H.; Ireland-Ripert, J.; Mourel, R. M. Analusis 1993, 21, 287-291.

5. Wolf, W. R.; Andrews, K. W. Fresenius J. Analytical Chemistry 1995, 352, 73.

6. Cornell, J. A. Experiments with Mixture - Designs, Models and Analysis of Mixture Data, Second Edition, A WileyInterscience Publication, John Wiley \& Sons, Inc., 1990.

7. Feinberg, M. H.; Favier, J. C.; Ireland-Ripert, J. Répertoire Général des Aliments; Paris: Lavoisier Technique and Documentation, 1991.
8. Statgraphics ${ }^{\circledR}$ Plus, Reference Manual, Version 1.4. Manugistics Inc., Cambridge, MA, 1994.

9. Droesbeke, J.-J.; Fine, J.; Saporta, G. Plans d'Expériences Applications à l'Entreprise; Paris: Éditions Technip, 1997, pp 279-309.

10. Rutledge, D. N. A Windows Program for Relaxation Parameter Estimation, in: Rutledge, D. N. (Ed.), Signal Treatment and Signal Analysis in NMR; Amsterdam: Elsevier, 1996, pp 191217.

11. Marquardt, D. W. J. Soc. Ind. Appl. Math. 1963, 11, 431-441. 